\title{
The measurement frequency and completeness of vital signs in general hospital wards: an evidence free zone
}

\author{
Gary B Smith, FRCA, FRCP, \\ University of Bournemouth, Faculty of Health and Social Sciences, Christchurch Road, \\ Bournemouth, Dorset, BH1 3LT, United Kingdom \\ gbsresearch@virginmedia.com
}

\begin{abstract}
Alejandra Recio-Saucedo, BSc, MSc, PhD,
University of Southampton, Centre for Innovation and Leadership in Health Sciences, National Institute for Health Research (NIHR) Collaboration for Applied Health Research and Care (CLAHRC) Wessex, Southampton, United Kingdom, University Road, Nightingale Building, Highfield Campus, Southampton SO17 1BJ +44 (23) 80597971

A.Recio-Saucedo@soton.ac.uk

Peter Griffiths, BA PhD RN,

University of Southampton, Centre for Innovation and Leadership in Health Sciences, National Institute for Health Research (NIHR) Collaboration for Applied Health Research and

Care (CLAHRC) Wessex, Southampton, United Kingdom, University Road, Nightingale Building, Highfield Campus, Southampton SO17 1BJ

peter.griffiths@soton.ac.uk
\end{abstract}

\section{Corresponding author:}

Professor G B Smith, FRCA, FRCP, Centre of Postgraduate Medical Research \& Education (CoPMRE), Faculty of Health and Social Sciences, Bournemouth University, Royal London House, Christchurch Road, Bournemouth, Dorset BH1 3LT, United Kingdom

Tel: +44 (0) 1202962782

Fax: +44 (0) 1202962218

Email: gbsresearch@virginmedia.com

Word count $=1869 \quad$ References $=59$ 
Measuring a patient's vital signs in hospital is fundamental to clinical assessment, risk evaluation and to preventing patient deterioration. It contributes significantly to routine nursing workload $(1,2)$ on general hospital wards, as most intermittent monitoring is manual, even when using electronic equipment. Despite the significance of this activity for patients and the work of nurses, the extent to which current practice is evidence based is questionable.

Among others, the frequency and content of vital sign datasets on general wards depend upon tradition (3), national guidance and consensus statements (4-7), the patient's diagnosis, clinical leadership and decision-making, and clinical workload (8-10). There is some agreement in published guidance regarding how often measurements should be taken and what parameters should always be measured, however much of this is based upon existing custom or perceived best practice rather than evidence (4-7). A 12-hourly minimum frequency is used in the UK, where many hospitals also use an aggregate weighted score derived from vital sign measurements (i.e., an early warning score) to determine the timing of subsequent observation sets $(4,5)$. Hospitals in other countries use different routine frequency regimens with some recommending measurement as often as 2-hourly $(6,11-14)$. Guidance often advises the routine recording of heart rate, blood pressure, respiratory rate, temperature, pulse oximetry, and level of consciousness $(6,7)$. Others additionally recommend the routine measurement of inspired oxygen concentration (5) or urine output (15) to calculate an early warning score value.

The potential to alter clinical outcomes depends upon the ability to detect and recognize an acute change in a patient's physiology. Intuitively, therefore, higher vital sign measurement frequencies and more complete observations sets should increase the probability that deterioration will be detected early. Some published research results support this. Australian researchers have reported significant reductions in unplanned ICU admissions and unexpected hospital deaths, when the mean daily vital sign measurement frequency increased from 3.4 to 4.5 times per day (16). A study from the Netherlands showed that protocolised vital signs measurement (i.e., three times daily) resulted in better detection of physiological abnormalities and more reliable activation of a rapid response team than when 
undertaken only when 'clinically indicated' (17). Belgian researchers report that the use of a standard nurse observation protocol, in which increased sickness (calculated from the vital signs using an early warning score) led to an increased vital sign frequency, reduced hospital length of stay and mortality in postoperative patients (18), and a decrease in the number of serious adverse events for patients discharged from the ICU (19). Others suggest that a protocolised 'once a day' early warning score assessment may be sufficient to screen for major adverse events in hospital populations (20) and research from Denmark showed that, for low risk patients, 8-hourly was no better than 12-hourly measurement for reducing clinical deterioration (21). On the other hand, a recent review of monitoring techniques found that continuous patient monitoring allowed earlier detection of deterioration in general ward patients than 'usual care' and improves rapid response team activation (22). However, the authors concluded that there was insufficient evidence of effectiveness to support the routine adoption of continuous vital signs monitoring in general wards (22).

Current evidence suggests that compliance with vital sign monitoring protocols is often poor, with incomplete and infrequent observation sets $(2,15,23-33)$. Hands et al. reported that the daily pattern of vital sign documentation is not uniform, with large morning and evening peaks. This suggests batching of measurements in line with nursing schedules rather than individual patient severity (25). Fewer vital signs were documented at night compared with daytime, and re-assessments were often omitted at night even when the patient was obviously ill (25). These findings mirror practices reported by the UK National Patient Safety Agency, which concluded that observations are seen as low priority tasks and staff rarely carry out routine observations during the night (24). In contrast, Yoder et al. found that approximately $50 \%$ of nighttime measurements occurred in low-risk patients and that the frequency of measurements was unrelated to the patient's early warning score value (13).

Identifying interventions that might improve monitoring compliance would seem to be a crucial quality improvement step with the potential to improve clinical outcomes. For instance, adherence to national guidance and local protocols/policies (4-7) provides goals against which practice can be compared. Regular audits of practice with personal feedback regarding 
compliance can serve to identify deficiencies and guide quality improvement measures (34, 35), especially if there is a focus on poorly performing wards (34). The establishment of local clinical champions also seems important (34). However, these need to be accompanied by staff education regarding monitoring protocols and their importance $(34,35)$. Clinical hierarchies, professional culture, prior experience of responses received to reporting abnormal vital signs, lack of confidence and staff opinion about the usefulness of vital signs monitoring or early warning score systems appear to be crucial in influencing adherence to monitoring policies $(2,24,36)$.

Despite evidence for the benefits of protocols for observation based on early warning scores the choice of vital signs for measurement and the subsequent clinical actions often appear to rely solely on nurses' clinical judgement or time availability, rather than on policies (2). There is also evidence that intuition plays an important part in nurses' detection of deterioration, and vital signs are used to validate intuitive feelings (37). Therefore, organisations need to reinforce good practice based on an understanding of human factors to remove barriers to compliance (34). The introduction of standardised observation and escalation protocols has been shown to increase the frequency of vital sign measurement $(17,18,21,34,38)$. Applying consistent policies across an organization is also likely to reduce the potential for errors in monitoring practice. Ensuring a clear, documented vital signs measurement plan for each patient with unambiguous instructions regarding the variables to be measured and the frequency of measurement is essential (4-7). Ideally, vital sign datasets should also be complete every time they are collected; otherwise, physiological instability can be missed (39). Senior staff should always be involved in decisions to reduce monitoring frequency below recommended levels $(4,7)$.

The presence of sufficient equipment to measure vital signs and its proper functioning are crucial to staff's ability to comply with vital signs protocols. There is a clear association between low levels of monitoring equipment, and wards' abilities to undertake observations to an agreed 4-hourly target and to increase the assessment frequency as advised by an early warning score policy (34). Similarly, clinical resource issues (e.g., clinical staffing, workload, 
patient turnover) and competing nursing activities also impact on the ability of wards to achieve vital sign compliance $(9,10,34)$. Although there are no data to support a minimum number of nurses on duty at any one time for this purpose, evidence indicates that an increase in nurses' workload increases the likelihood of inpatient hospital deaths (40) and of nursing activities related to surveillance remaining undone (41). Therefore, specific research is required to understand (a) the relationship between nurse staffing, workload, vital signs monitoring and outcomes, and (b) the reasons behind the apparent reduction in patient monitoring at night.

Introducing an early warning score system has been shown to increase the respiratory rate recording on general wards $(33,42)$. However, as the early warning score value dictates when the next set of vital signs is due, errors in early warning score calculation may affect both the frequency at which vital signs are measured and the resulting staff workload $(43,44)$. Similarly, early warning score systems with poor sensitivity and specificity, and poorly designed escalation protocols, may also have an influence on the number of escalations, which, in time, may produce alarm fatigue (45). This could impact on future compliance with monitoring protocols. Understanding staff attitudes towards early warning score systems is also crucial in ensuring their appropriate and correct use. For instance, Bunkenborg et al. reported that nursing staff adhered less to an early warning score algorithm in patients with higher total early warning score values that called for more frequent measurements (38). Likewise, interviewees in a study by Skyttberg et al. described the perceived importance of following a standardized vital sign measurement process, but felt that the standard might not be regarded as relevant where patients had minor conditions. They also reported that experienced staff were more likely to deviate from the standard (35).

The introduction of a rapid response team appears to lead to an increase in vital signs monitoring $(42,46)$, as do the design and standardization of vital signs charts (47-51). Paterson et al. found that vital signs, particularly respiratory rate and conscious level, were more completely documented after introducing a standardised early warning score chart (47). Elliott et al. investigated the impact on monitoring of a range of different charts and found that 
compliance improved (48). Several studies have shown that the combination of a new observation chart and associated training led to improved completeness $(16,49-51)$ and frequency (51) of vital sign sets. However, others have shown no significant improvement in the frequency of vital signs sets, completeness of observations or rechecking of vital signs in line with an early warning score protocol (52-55).

Technology may have a role to play in improving patient monitoring. For instance, methods known to reduce the time taken to document vital signs in the patient's record (e.g., mobile devices) might be expected to free up clinical time making it possible for staff to document more observations (56). Although use of automated continuous monitoring appears to be an attractive approach to increasing the frequency of vital sign monitoring in general wards, currently, not all components of early warning score can be measured continuously and automatically. Continuous monitoring is also currently costly and it is unclear whether its hospital-wide implementation would be beneficial (22). Electronic health records do not necessarily improve the documentation of vital signs (57). Skyttberg et al. studied vital sign data quality, including completeness in electronic health records used in Swedish emergency departments (35) and some of their findings seem transferable to general hospital wards. Interviewees believed that well-designed information systems that facilitated vital sign measurement and documentation, and allowed vital signs to be viewed at the bedside were useful in improving practice (35). Such technology facilitates accurate summation of the early warning score and appears to improve staff adherence to requests to taking of observations at predefined time points $(58,59)$.

Despite being amongst the most common hospital practices, many questions remain unanswered regarding vital signs monitoring. Current practice cannot be said to be evidence based. Little is known about which vital signs should be measured routinely and at what frequency predominantly because evidence regarding the timing, patterns, and rates of deterioration in different patients and conditions is sparse. Whilst there are still uncertainties about how often vital signs should be measured and what parameters should always be measured, it would appear that improved patient monitoring improves some patient outcomes 
and yet compliance with established protocols remains poor. There are opportunities to improve the frequency and content of vital signs datasets and some evidence regarding which interventions may be useful. However, properly designed, prospective studies are urgently required to measure the impact of individual or grouped interventions on measurement compliance and clinical outcomes. 


\section{References}

1. Yeung MS, Lapinsky SE, Granton JT, Doran DM, Cafazzo JA. Examining nursing vital signs documentation workflow: barriers and opportunities in general internal medicine units. Journal of Clinical Nursing, 2012; 21: 975-982.

2. Cardona-Morrell M, Prgomet M, Lake R, Nicholson M, Harrison R, Long J, Westbrook J, Braithwaite J, Hillman K. Vital signs monitoring and nurse-patient interaction: A qualitative observational study of hospital practice. International Journal of Nursing Studies. 2016; 56: 9-16.

3. Zeitz K, McCutcheon H. Evidence-based practice: to be or not to be, this is the question! Int J Nurs Pract. 2003; 9: 272-279.

4. National Institute for Health and Clinical Excellence: Acutely ill patients in hospital: recognition of and response to acute illness in adults in hospital. NICE clinical guideline No.50. London; 2007.

5. National Early Warning Score (NEWS): Standardising the assessment of acute-illness severity in the NHS. Report of a working party. Royal College of Physicians, London, 2012.

6. Australian Commission on Safety and Quality in Health Care (2010). National Consensus Statement: Essential Elements for Recognising and Responding to Clinical Deterioration. Sydney, ACSQHC.

7. DeVita MA, Smith GB, Adam S, Buist S, Bellomo R, Bonello R, Cerchiari E, Farlow B, Goldsmith D, Haskell H, Hillman K, Howell M, Hravnack M, Hunt E, Hvarfner A, Kellett J, Lighthall GK, Lippert A, Lippert F, Mahroof R, Myers J, Pizaro I, Rosen M, Reynolds S, Rotondi A, Rubulotta F, Winters B. Consensus Conference on the Afferent Limb: Identifying Hospitalised Patients in Crisis. Resuscitation 2010; 81: 375-82.

8. Hogan J. Why don't nurses monitor the respiratory rates of patients? British Journal of Nursing, 2006; 15: 489-492

9. Mok W, Wang W, Cooper S, Ang EN, Liaw SY. Attitudes towards vital signs monitoring in the detection of clinical deterioration: scale development and survey of ward nurses. Int J Qual Health Care. 2015; 27: 207-13. 
10. Mok WQ, Wang W, Liaw SY. Vital signs monitoring to detect patient deterioration: An integrative literature review. Int J Nurs Pract. 2015; 21 Suppl 2: 91-8.

11. Hammond NE, Spooner AJ, Barnett AG, Corley A, Brown P, Fraser JF. The effect of implementing a modified early warning scoring (MEWS) system on the adequacy of vital sign documentation. Aust Crit Care 2013; 26: 18-22.

12. Tanriover MD, Yildirim G, Kehya E, Erdogan O, Nacar DK, Ozisik L, Topeli A. Does the implementation of modified early warning scores spare workforce by decreasing the frequency of nurse assessments? Acta Medica 2014; 3: 80-83.

13. Yoder JC, Yuen TC, Churpek MM, Arora MV, Edelson DP. A Prospective Study of Nighttime Vital Sign Monitoring Frequency and Risk of Clinical Deterioration. JAMA Intern Med. 2013; 173: 1554-1555.

14. Schulman CS, Staul L. Standards for Frequency of Measurement and Documentation of Vital Signs and Physical Assessments. Crit Care Nurse 2010; 30: 74-76

15. Gordon CF, Beckett DJ. Significant deficiencies in the overnight use of a Standardised Early Warning Scoring system in a teaching hospital. Scottish Medical Journal 2011; 56: $15-18$.

16. Mitchell IA, McKay $\mathrm{H}$, van Leuvan $\mathrm{C}$ et al. A prospective controlled trial of the effect of a multifaceted intervention on early recognition and intervention in deteriorating hospital patients. Resuscitation 2010; 81: 658-66.

17. Ludikhuize J, Borgert M, Binnekade J, Subbe C, Dongelmans D, Goossens A. Standardized measurement of the Modified Early Warning Score results in enhanced implementation of a rapid response system: a quasi-experimental study. Resuscitation 2014; 85: 676-82.

18. De Meester K, Haegdorens F, Monsieurs KG, Verpooten GA, Holvoet A, van Bogaert P. Six-day postoperative impact of a standardized nurse observation and escalation protocol: a preintervention and postintervention study. J Crit Care 2013; 28: 1068-74.

19. De Meester K, Das T, Hellemans K, Verbrugghe W, Jorens PG, Verpooten GA, et al. Impact of a standardized nurse observation protocol including MEWS after Intensive Care Unit discharge. Resuscitation 2013; 84: 184-8. 
20. van Galen LS, Dijkstra CC, Ludikhuize J, Kramer MHH, Nanayakkara PWB. A Protocolised Once a Day Modified Early Warning Score (MEWS) Measurement Is an Appropriate Screening Tool for Major Adverse Events in a General Hospital Population. PLoS ONE. 2016;11:e0160811.

21. Petersen JA, Antonsen Kb, Rasmussen LS. Frequency of early warning score assessment and clinical deterioration in hospitalized patients: A randomized trial. Resuscitation. 2016; 101: 91-96.

22. Cardona-Morrell M, Prgomet M, Turner RM, Nicholson M, Hillman K. Effectiveness of continuous or intermittent vital signs monitoring in preventing adverse events on general wards: a systematic review and meta-analysis. Int J Clin Pract. 2016; 70: 806-824.

23. National Confidential Enquiry into Patient Outcomes and Death. "An acute problem?" London: National Confidential Enquiry into Patient Outcome and Death; 2005.

24. National Patient Safety Agency (2007) Recognising and responding appropriately to early signs of deterioration in hospitalised patients. NPSA, London.

25. Hands C, Reid E, Meredith P, Smith GB, Prytherch DR, Schmidt PE, Featherstone PI. Patterns in the recording of vital signs and early warning scores - time of day, day of week. BMJ Quality \& Safety. 2013; 22: 719-726.

26. Sundararajan K, Flabouris A, Thompson C, Seppelt I, on behalf of the George Institute of Global Health and the Australian and New Zealand Intensive Care Society Clinical Trials Group Point Prevalence study Investigators. Hospital overnight and evaluation of systems and timelines study: A point prevalence study of practice in Australia and New Zealand. Resuscitation. 2016; 100: 1-5.

27. Armstrong B, Walthall $\mathrm{H}$, Clancy $\mathrm{M}$, Mullee $\mathrm{M}$, \& Simpson $\mathrm{H}$. Recording of vital signs in a district general hospital emergency department. Emergency Medicine Journal. 2008; 25: 799-802.

28. Johnson KD, Winkelman C, Burant C, Dolansky M, Totten V. (2012). The factors that affect the frequency of vital sign monitoring in the emergency department. Journal of Emergency Nursing. 2014; 40: 27-35.

29. Van Leuvan $\mathrm{CH}$, Mitchell I. Missed opportunities. An observational study of vital sign measurements. Crit Care Resusc. 2008; 10: 111-5. 
30. Kyriacos U, Jelsma J, Jordan S (2014) Record Review to Explore the Adequacy of PostOperative Vital Signs Monitoring Using a Local Modified Early Warning Score (Mews) Chart to Evaluate Outcomes. PLoS ONE 9(1): e87320. doi:10.1371/journal.pone. 0087320

31. Asiimwe SB, Okello S, Moore CC (2014) Frequency of Vital Signs Monitoring and its Association with Mortality among Adults with Severe Sepsis Admitted to a General Medical Ward in Uganda. PLoS ONE 9(2): e89879. doi:10.1371/journal.pone.0089879

32. McGain F, Cretikos MA, Jones D, Van Dyk S, Buist MD, Opdam H, Pellegrino V, Robertson MS, Bellomo R. Documentation of clinical review and vital signs after major surgery. Med J Aust 2008; 189: 380-383.

33. McBride J, Knight D, Piper J, Smith GB. Long-term effect of introducing an early warning score on respiratory rate charting on general wards. Resuscitation. 2005; 65: 41-4.

34. Wood SD, Candeland JL, Dinning A, Dow S, Hunkin H, McHale S, McNeill G, Taylor N. Our approach to changing the culture of caring for the acutely unwell patient at a large UK teaching hospital: A service improvement focus on Early Warning Scoring tools. Intensive and Critical Care Nursing. 2015; 31: 106-115.

35. Skyttberg N, Vicente J, Chen R, Blomqvist H, Koch S. How to improve vital sign data quality for use in clinical decision support systems? A qualitative study in nine Swedish emergency departments. BMC Medical Informatics and Decision Making. 2016; $16: 61$.

36. Osborne S, Douglas C, Reid C, Jones L, Gardner G. The primacy of vital signs - acute care nurses' and midwives' use of physical assessment skills: a cross sectional study. Int. J. Nurs. Stud. 2015;52: 951-962.

37. Odell $\mathrm{M}$, Victor $\mathrm{C}$, Oliver $\mathrm{D}$. Nurses' role in detecting deterioration in ward patients: systematic literature review. J Adv Nurs. 2009;65:1992-2006.

38. Bunkenborg G, Poulsen I, Samuelson K, Ladelund S, Åkeson J.Mandatory early warning scoring -implementation evaluated with a mixed-methods approach. Applied Nursing Reseach 2016; 29: 168-176.

39. Clifton DA, Clifton L, Sandu DM, Sm,ith GB, Tarassenko L, Vollam SA, Watkinson PJ. 'Errors' and omissions in paper-based early warning scores: the association with changes in vital signs—a database analysis. BMJ Open. 2015;5: e007376. 
doi:10.1136/bmjopen-2014-007376.

40. Griffiths P, Ball J, Drennan J, et al. Nurse staffing and patient outcomes: Strengths and limitations of the evidence to inform policy and practice. A review and discussion paper based on evidence reviewed for the National Institute for Health and Care Excellence Safe Staffing guideline development. Int J Nurs Stud 2016;63:213-25.

41. Jones TL, Hamilton P, Murry N. Unfinished nursing care, missed care, and implicitly rationed care: State of the science review. Int J Nurs Stud. 2015; 52: 1121-37.

42. Odell M, Rechner IJ, Kapila A, Even T, Oliver D, Davies CWH, Milsom L, Forster A, Rudman K. The effect of a critical care outreach service and an early warning scoring system on respiratory rate recording on the general wards. Resuscitation. 2007; 74: 470475 .

43. Kolic I, Cranea S, McCartney S, Perkins Z, Taylor A. Factors affecting response to National Early Warning Score (NEWS). Resuscitation. 2015; 90: 85-9.

44. Watson A, Skipper C, Steury R, Walsh H, Levin A. Inpatient nursing care and early warning scores: a workflow mismatch. J Nurs Care Qual. 2014; 29: 215-22.

45. Sendelbach S, Funk M. Alarm fatigue: a patient safety concern. AACN Adv Crit Care. 2013; 24: 378-86

46. Chen J, Hillman K, Bellomo R, Flabouris A, Finfer S, Cretikos M, The MERIT Study Investigators for the Simpson Centre and the ANZICS Clinical Trials Group. The impact of introducing medical emergency team system on the documentations of vital signs. Resuscitation 2009; 80: 35-43.

47. Paterson R, MacLeod DC, Thetford D, et al. Prediction of in-hospital mortality and length of stay using an early warning scoring system: clinical audit. Clin Med 2006; 6: 281-4.8.

48. Elliott D, Emily Allen E, McKinley S, Perry L, Duffield C, Fry M, GallagherR, ledema R, Roche M. User compliance with documenting on a track and trigger-based observation and response chart: a two-phase multi-site audit study. Journal of Advanced Nursing 2017 DOI: 10.1111/jan.13302

49. Hammond NE, Spooner AJ, Barnett AG, Corley A, Brown P, Fraser JF. The effect of implementing a modified early warning scoring (MEWS) system on the adequacy of vital sign documentation. Australian Critical Care. 2013; 26: 18-22. 
50. Cahill $\mathrm{H}$, Jones $\mathrm{A}$, Herkes $\mathrm{R}$ et al. Introduction of a new observation chart and education programme is associated with higher rates of vital-sign ascertainment in hospital wards. BMJ Qual Saf 2011; 20: 791-6.

51. Kyriacos U, Jelsma J, James M, Jordan S. Early warning scoring systems versus standard observations charts for wards in South Africa: a cluster randomized controlled trial. Trials. 2015; 16: 103.

52. Fernandez R, Griffiths R. A comparison of an evidence based regime with the standard protocol for monitoring postoperative observation: a randomized controlled trial. Aust $\mathrm{J}$ Adv Nurs 2005; 23: 15-21.

53. Robb G, Seddon M. A multi-faceted approach to the physiologically unstable patient. Qual Saf Health Care 2010; 19: e47.

54. Benning A, Ghaleb M, Suokas A et al. Large scale organisational intervention to improve patient safety in four UK hospitals: mixed method evaluation. BMJ 2011; 342: d195.

55. Benning A, Dixon-Woods M, Nwulu U et al. Multiple component patient safety intervention in English hospitals: controlled evaluation of second phase. BMJ 2011; 342: d199.

56. Meccariello M, Perkins D, Quigley LG, Rock A, Qiu J. Vital Time Savings. Evaluating the use of an automated vital signs documentation system on a medical/surgical unit. JHIM 2010; 24: 46-51.

57. Stevenson JE, Israelsson J, Nilsson GC, Petersson GI, Bath PA. Recording signs of deterioration in acute patients: The documentation of vital signs within electronic health records in patients who suffered in-hospital cardiac arrest. Health Informatics J. 2016; 22: 21-33.

58. Jones S, Mullaly M, Ingleby S, Buist M, Bailey M, Eddleston JM. Bedside electronic capture of clinical observations and automated clinical alerts to improve compliance with an Early Warning Score protocol. Crit Care Resusc. 2011;13:83-8.

59. Mohammed M, Hayton R, Clements G, Smith G, Prytherch D. Improving accuracy and efficiency of early warning scores in acute care. Br J Nurs. 2009;18:18-24. 